\title{
SHORT WAVE BREAKING EFFECTS ON LOW FREQUENCY WAVES
}

\author{
Christopher Daly ${ }^{*}, 1,2$, Dano Roelvink ${ }^{1,2,3}$, Ap van Dongeren ${ }^{1}$, \\ Jaap van Thiel de Vries ${ }^{1,2}$, and Robert McCall ${ }^{1}$
}

\begin{abstract}
The effect of short wave breaking on low frequency waves is investigated using two breaker formulations implemented in a time-dependent numerical model (XBeach): (1) an advective-deterministic approach (ADA) and (2) the probabilistic breaker formulation of Roelvink (1993). Previous research has shown that the ADA breaker model gives different results for the cross-shore pattern of the fraction of breaking waves, which is now shown to affect not only the short wave height but also the short wave groupiness. While RMS short wave heights are comparable to measurements using both breaker models, the ADA breaker model allows higher levels of short wave groupiness into the surf zone. It is shown that this acts as an additional forcing mechanism for low frequency waves in the shoaling and nearshore zone, which, in addition to greater levels of breaking, leads to higher values of wave set-up. These findings are dependent on the complexity of the local bathymetry. For a plane slope, the differences in the low frequency wave heights and set-up predicted by both breaker models are negligible. Where arbitrary breakpoints are present in the field of wave propagation, such as nearshore bars or reefs, the ADA model predicts higher localized set-up, indicative of greater flow over such features. Differences are even more pronounced when the incident wave regime is highly energetic.
\end{abstract}

Keywords: wave breaking; low frequency waves; fraction of breaking waves; wave set-up; wave groupiness; XBeach

\section{INTRODUCTION}

\section{Background}

Low Frequency (LF) waves, also commonly referred to as long waves, infragravity waves and surf beat, were discovered some sixty years ago by Munk (1949), who observed LF motions outside the surf zone and suggested that it was caused by variations in the mass transport of groups or 'beats' of incident waves. Many recent studies (e.g. Raubenheimer and Guza 1996, van Thiel de Vries 2006) have found that LF wave energy dominates the wave spectrum in the inner surf zone and that LF waves are able to run-up farthest on a beach. LF wave run-up therefore plays an integral role in beach morphology, especially during storms where their nearshore amplitudes are highest.

Forced LF waves formed as a result of groups of short waves (Longuet-Higgins and Stewart, 1962) are released during the short wave breaking process (c.f. Battjes et al., 2004). The short wave breaking process also generates free LF wave motions. Symonds et al. (1982) and Schaffer and Svendsen (1988) introduced two concepts for the generation of free LF waves by a moving and fixed breakpoint concept respectively. The free LF waves generated by this mechanism are able to move both shoreward and seaward of the mean breakpoint on the timescale of wave groups. A hybrid model is later presented by Schäffer (1993) which combines the first two free LF wave generation mechanisms, hence variation of the initial breakpoint position while allowing wave groupiness into the surf zone.

The correct physical representation of the short wave breaking process is therefore a key factor in determining combined bound and free LF wave motions in the surf zone. Wave breaking formulations must therefore be able to describe the pattern and frequency of wave breaking across the surf zone over a range of conditions. Most wave breaking formulations assign a probabilistic distribution to account for wave breaking occurrence which is dependent on the local wave height, water depth and a breaker parameter. If these models are applied to varied nearshore bathymetry, for example a nearshore bar or step, sudden changes in the water depth will lower the probability of breaking, $Q_{b}$, to values close to zero, which can be interpreted as the instantaneous secession of breaking. Contradictory to this is that in nature waves tend to break for a given period of time after the process is initiated before stabilizing and reforming again if the surf zone is unsaturated.

\section{Objectives}

In this paper, we investigate two short wave breaking formulations applicable to wave-groupforced surf beat models in order to determine their effectiveness in not only representing timeaveraged, cross-shore, RMS short wave heights, but also the LF wave heights and wave set-up for various surf zone conditions and nearshore topographies. As the base case, the breaker formulation of

\footnotetext{
* Now at: MARUM, Universität Bremen, Leobener Strasse, D-28359 Bremen, Germany

${ }^{1}$ Deltares, P.O.Box 177, 2600 MH Delft, the Netherlands

${ }_{2}^{2}$ Civil Engineering and Geosciences, Delft University of Technology, Stevinweg 1, 2627 CN Delft, the Netherlands

${ }^{3}$ Water Engineering, UNESCO-IHE Institute for Water Education, Westvest 7, 2611 AX Delft, the Netherlands
} 
Roelvink (1993) (Rv93 hereafter) is used. This is compared to an advective-deterministic approach (ADA hereafter) to wave dissipation through breaking (Daly et al., in review). In the ADA breaker model, the state of breaking is advected shoreward until it is turned on $\left(Q_{b}=1\right)$ or off $\left(Q_{b}=0\right)$ by specifying upper and lower values of the breaker parameter, $\gamma_{b}$ and $\gamma_{r}$ respectively, with the lower termed "wave reforming" after Dally et al. (1984) and Dally (1990), who used the concept to model a wave-by-wave approach to breaking.

In the Rv93 formulation, wave breaking is described in probabilistic terms whereas in the ADA formulation breaking is deterministic. Both breaker models are implemented in the time-dependent, wave-group-forced numerical model, XBeach (Roelvink et al., 2009). Three flume experiments each with three test cases are used for the study. Each experiment features a different type of fixed bathymetry; a plane, a bar-trough and a stepped slope.

\section{NUMERICAL MODEL}

XBeach (Roelvink et al. 2009) is a wave-group-forced, time-varying, depth-averaged, surfbeat morphological model designed to include the influence of LF waves in nearshore hydrodynamics during storms events. It is used in the present study to compare the two wave breaking formulations (Rv93 and the ADA). XBeach consists of different modules: a short wave module (including the proposed wave breaking schemes) and flow module (driven by the non-linear shallow water equations NSWE) together forms the core hydrodynamic part of the numerical model. A brief description of these XBeach modules is given below. For full details, the reader is referred to Roelvink et al. 2009.

\section{Short Wave Action Balance Equations}

An input short wave-group timeseries is defined in terms of the wave action and modeled using the time-varying wave action balance equations, including wave shoaling and refraction, given onedimensionally as:

$$
\begin{gathered}
\frac{\partial A}{\partial t}+\frac{\partial c_{g} A}{\partial x}=-\frac{D_{t o t}}{\sigma} \\
\text { and } A(x, t)=\frac{E(x, t)}{\sigma(x, t)}
\end{gathered}
$$

where: $A=$ wave action in space $(x)$ and time $(t) ; \sigma=$ intrinsic wave frequency (from linear theory); $c_{g}$ $=$ wave group celerity (from linear theory); $E=$ wave energy (which varies on the wave group timescale); and $D_{t o t}=$ total dissipation (which accounts for frictional dissipation $\left(D_{f}\right)$ and wave breaking dissipation $\left(D_{w}\right)$, described further below). The roller dissipation model of Deigaard (1993) is included in the wave dissipation terms (c.f. Roelvink et al. 2009 for full details).

\section{Shallow Water Equations}

XBeach couples the short wave module (as given above) and the non-linear shallow water equations (NSWE) in order to compute the LF surface elevations. Radiation stress gradients associated with the short waves is used as the forcing term for the NSWE. The (one-dimensional) depth-averaged and short wave-averaged shallow water equations are given as:

$$
\begin{gathered}
\frac{\partial u}{\partial t}+u \frac{\partial u}{\partial x}-v_{h}\left(\frac{\partial^{2} u}{\partial x^{2}}\right)=\frac{F_{x}}{\rho h}-\frac{\tau_{b x}}{\rho h}-g \frac{\partial \eta}{\partial x} \\
\text { and } \frac{\partial \eta}{\partial t}+\frac{\partial h u}{\partial x}=0
\end{gathered}
$$

where: $h=$ water depth; $u=$ LF Lagrangian velocity; $\tau_{b x}=$ bed shear stress; $g=$ acceleration of gravity; $\rho=$ water density; $v_{h}=$ horizontal eddy viscosity; $\eta=$ water level; and $F_{\mathrm{x}}=$ short wave-induced stress.

\section{Wave Breaking Formulations}

The breaker model of Rv93 is a bore-based wave dissipation formulation which is used together with the wave action balance equation in (1). The wave energy dissipation is given as:

$$
D_{w}=P_{b} D_{b}
$$




$$
\begin{gathered}
\text { given } D_{b}=\frac{\alpha}{4} \rho g f H_{b}^{2} \\
\text { and } P_{b}=1-\exp \left[-\left(\frac{H}{\gamma h}\right)^{n}\right] \\
\text { where } H_{b}=\gamma h
\end{gathered}
$$

and where: $D_{b}=$ dissipation due to wave breaking; $P_{b}=$ 'weighting function' or probability of breaking; $\alpha=$ intensity of wave dissipation; $f=$ intrinsic wave frequency; $H=$ instantaneous RMS wave height; $\mathrm{n}=$ coefficient; $\gamma=$ breaker coefficient; and $H_{b}=$ height of the wave at breaking (assumed to be in the same order as the water depth $\approx 1$ ).

The ADA breaker model (Daly et al., in review) is also a bore-based wave dissipation formulation which makes use of equations 5 and 6; however, the representation of wave breaking is deterministic. This is done by changing the definition of the probability function (in equation 7) to represent the 'state of wave breaking' (renamed $B$ for clarity) being either on (wave is breaking, hence $B=1$ ) or off (wave is not breaking, hence $B=0$ ). In doing so, wave breaking is tracked from the assumed point where it starts (when $H>\gamma_{b} h$; where: $\gamma_{b}=$ breaker parameter) until the point where it stops (when $H<\gamma_{r} h$; where: $\gamma_{r}=$ reformation parameter). $\gamma_{b}$ and $\gamma_{r}$ are expressed as:

$$
\gamma_{b}=\frac{H_{b}}{h} \text { and } \gamma_{r}=\frac{H_{r}}{h}
$$

Therefore, for values of the instantaneous ratio of $H / h$ between the upper and lower limits of $\gamma_{b}$ and $\gamma_{r}$ respectively, $B$ is determined by and, additionally, advected with the speed of the individual wave, $c$, as follows:

$$
\begin{gathered}
B=1, H>\gamma_{b} h \\
\frac{\partial B}{\partial t}+c \frac{\partial B}{\partial x}=0, \gamma_{r} h<H<\gamma_{b} h \\
B=0, H<\gamma_{r} h
\end{gathered}
$$

The advective component of the formulation (in equation 11) ensures that the history of wave breaking is maintained as $B$ is propagated shoreward, which therefore does not lead to premature switching off of wave breaking once it has begun. Daly et al. (in review) carried out a calibration and validation study of the ADA breaker model to evaluate its performance in predicting cross-shore wave heights and fraction of breaking waves. Optimized values of 0.52 and 0.30 were obtained for $\gamma_{b}$ and $\gamma_{r}$ respectively, which are also used in the present work.

The time-averaged fraction of breaking, $Q_{b}$, can be determined for either breaker model by simply finding the time-average of $P_{b}$ (Rv93) or $B$ (ADA) over the (long-term) simulation period. Despite differences in the definition of $P_{b}$ and $B$ between the Rv93 and ADA breaker models, $Q_{b}$ is a comparable parameter.

\section{EXPERIMENTAL SET-UP AND DATASETS}

Three flume experiments are considered in the investigation, each featuring a different type of bathymetry. Firstly the experiments of Boers (1996) (hereafter Boers) featuring a bar-trough nearshore bathymetry; secondly Van Noorloos (2003) (first presented in Van Dongeren et al., 2007; hereafter VN) featuring a plane slope; and finally, Van der Meer (1990) (hereafter VdM) featuring a stepped nearshore bathymetry. All of the experiments were carried out in the Fluid Mechanics Laboratory at Delft University of Technology and use irregular wave conditions. A brief description of the wave flume setup and the wave cases are given following. Depending on the experiment, only certain variables were measured or can be derived from the data. This is also mentioned below as well as the analytical techniques used to process the data. 


\section{Wave Flume Set-up}

The flume experiments were all conducted a $40 \mathrm{~m}$ long, $1.05 \mathrm{~m}$ deep and $0.8 \mathrm{~m}$ wide wave flume. The flume is equipped with a piston-type, second-order wave generator capable of active reflection compensation (ARC). In all of the experiments, the bottom surface level is fixed using smoothed concrete.

In the Boers experiments, the bathymetry is reproduced and scaled from the LIP 11D experiments carried out in the Delta Flume (Roelvink and Reniers, 1995) starting $5 \mathrm{~m}$ from the wave generator, which includes a bar-trough formation in the nearshore. In the VN experiments, a plane 1:35 slope is used starting $8 \mathrm{~m}$ from the wave generator. For the VdM experiments, a steep 1:15 slope beginning 9.7 $\mathrm{m}$ from the wave generator changes to a mild 1:260 slope $15 \mathrm{~m}$ from the wave generator.

A high spatial density of wave gauges are used for the Boers and VN experiments (typical spacing between 0.2 and $1.0 \mathrm{~m}$ ), while for the VdM experiment, a typical gauge spacing of $1.5 \mathrm{~m}$ is used. A deep water depth, $h_{0}$, (at the wave generator) of $0.75 \mathrm{~m}$ and $0.70 \mathrm{~m}$ is maintained for the Boers and $\mathrm{VN}$ experiments respectively, while for the VdM cases the still water level alternates from 0.56 to $0.66 \mathrm{~m}$. The bathymetry and location of wave gauges for all of the experiments are shown in Figure 1 below.
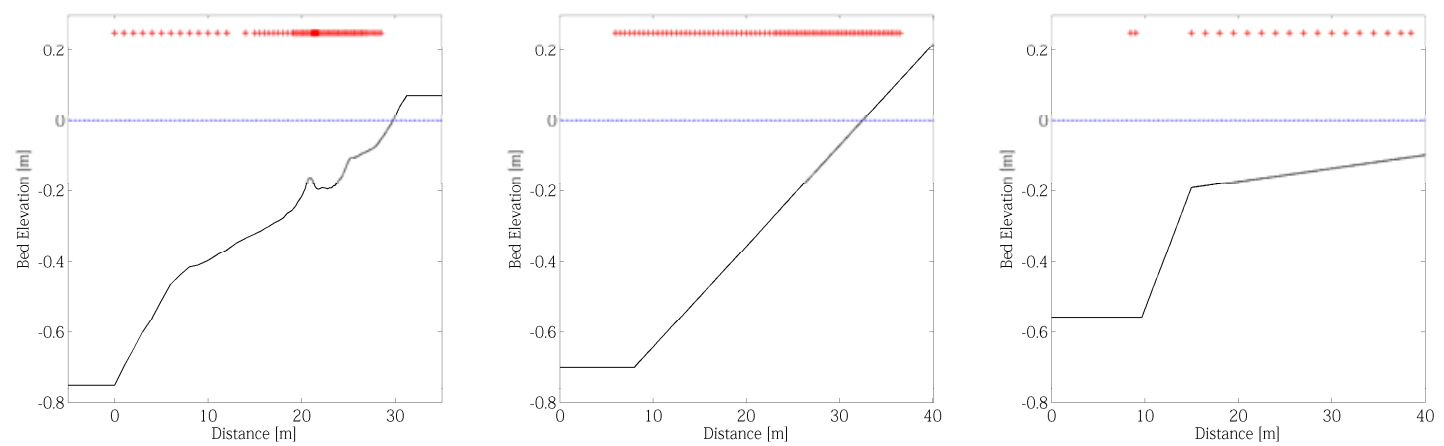

Figure 1: Bathymetry (solid line) and location of wave gauges (+) in the flume experiment of Boers (left), Van Noorloos (middle) and Van der Meer (right).

\section{Wave Cases}

For each of the experiments, the irregular wave cases are defined using a parameterized JONSWAP spectrum represented by a (spectral) significant wave height, $H_{m 0}$, and peak frequency, $f_{p}$. Boers used three wave conditions: case 1A, 1B and 1C. 1A and 1B have the highest $f_{p}$ values; however, $1 \mathrm{~B}$ has the highest $H_{m 0}$ value and therefore the most extreme wave steepness. Cases $1 \mathrm{~A}$ and $1 \mathrm{C}$ follow in decreasing order in terms of steepness, with the latter having the longest period waves.

VN conducted six irregular wave experiments of which we only use three cases, C1, C2 and C3. In these cases, $H_{m 0}$ is varied and $f_{p}$ is kept constant. The other cases of $\mathrm{VN}$ are similar to the ones selected, hence their exclusion.

VdM also conducted a number of experiments, but we will only use the cases TST015, TST110 and TST322 as they show the greatest dissipation of wave energy over the stepped bathymetry. Although TST322 has the steepest waves, the water depth in the flume is greater than in the other two cases. The details of all the irregular wave cases are given in Table 1 below.

\begin{tabular}{|c|c|c|c|c|c|}
\hline \multicolumn{7}{|c|}{ Table 1: Wave Case Parameters } \\
\hline Dataset & Case Name & $\boldsymbol{T}_{\boldsymbol{p}}(\mathbf{s})$ & $\boldsymbol{f}_{\boldsymbol{p}}(\mathbf{H z})$ & $\boldsymbol{h}_{\boldsymbol{0}}(\mathbf{m})$ & $\boldsymbol{H}_{\boldsymbol{m} \boldsymbol{}}(\mathbf{m})$ \\
\hline Boers & 1A & 2.10 & 0.476 & 0.75 & 0.160 \\
" & 1B & 2.10 & 0.476 & 0.75 & 0.220 \\
" & 1C & 3.40 & 0.294 & 0.75 & 0.107 \\
VN & C1 & 2.00 & 0.500 & 0.70 & 0.050 \\
" & C2 & 2.00 & 0.500 & 0.70 & 0.075 \\
VdM & C3 & 2.00 & 0.500 & 0.70 & 0.100 \\
" & TST015 & 2.28 & 0.438 & 0.56 & 0.071 \\
" & TST110 & 1.95 & 0.513 & 0.56 & 0.099 \\
& TST322 & 1.95 & 0.513 & 0.66 & 0.121 \\
\hline
\end{tabular}




\section{Measured and Derived Quantities}

The available dataset includes water surface elevation measurements for the Boers and VN experiments. The measured timeseries of water surface elevations are divided into short wave (high frequency) and LF components using Fast Fourier Transform (FFT) filtering. This is done in discrete frequency space, where a cut-off frequency, $f_{c u}$, marks the division between the short wave and LF ranges. The value of $f_{\text {cut }}$ is taken as is taken as half the value of the peak (short wave) frequency, $f_{p}$. (e.g. Van Dongeren et al. 2007). RMS short wave and LF wave heights are then obtained.

For the VdM experiments only processed RMS short wave height data was obtained and therefore no LF wave height data is available. The fraction of breaking waves is only measured during the Boers experiments.

\section{MODEL RESULTS}

Each of the nine cases shown in Table 1 was simulated using the XBeach numerical model. In this section, the results of five output variables from these simulations are presented for each bathymetric case. These are the:

1. Time averaged fraction of breaking waves $\left(Q_{b}\right)$

2. RMS short wave height $\left(H_{r m s}\right)$

3. Short wave groupiness factor $\left(G F_{H}\right)$

4. RMS LF wave height $\left(\zeta_{\mathrm{rms}}\right)$

5. Mean (time-averaged) water level $(M W L)$

Figure 2 through Figure 6 shows the results of the above five variables respectively for the nine cases considered. The $Q_{b}$ and $H_{r m s}$ results are already presented in Daly (in review), but are repeated here as they form the basis of the current investigation. The groupiness factor (List, 1992) is derived from the computed short wave height timeseries and is used to illustrate the groupiness as a function of the variance of the short waves as they shoal and break. This short wave groupiness factor is given as:

$$
G F_{H}=\frac{\sqrt{2} \cdot \sigma_{H}}{\bar{H}}
$$

where: $\sigma_{H}=$ standard deviation (of $H$ ); and $\bar{H}=$ time-average (of $H$ ). The $G F_{H}$ is not computed for the measured short wave height timeseries as the result would not be comparable to the computed data which is based on linear wave theory and therefore does not account for increased non-linear wave behavior inherent in the surf zone which is observed in the measurements.

The results for the five variables are systematically presented for each flume experiment (i.e. for Boers, then VN and then VdM). For brevity, the ADA breaker model results for each variable will be referred to as simply (variable)-ADA and, similarly, the Rv93 breaker model results will be presented as (variable)-Rv93. It is the intention to highlight differences between the two breaker models, however measured data is shown where available and is simply referred to as (variable)-Meas.

\section{Fraction of Breaking Waves}

Measured data in Figure 2 is shown only for the Boers wave cases. In these plots the level of wave breaking is quite low in the deeper section of the flume $\left(Q_{b}\right.$-Meas $\left.<0.2\right)$, but sharply increases at the crest of the nearshore bar. The higher levels of breaking $\left(Q_{b}\right.$-Meas $\left.>0.5\right)$ is sustained beyond the crest of the bar until at least the middle of the trough (the point of wave reformation for cases $1 \mathrm{~A}$ and 1B) and even further until the end of the trough (case 1C). A second sharp increase in $Q_{b}$-Meas occurs at the start of the inner surf zone at approximately $26 \mathrm{~m}$ in the flume. From this point up until the shoreline very high levels wave breaking persist $\left(Q_{b}\right.$-Meas $\left.>0.8\right)$. The dynamics of wave breaking are shown to be captured well by the ADA model, but contrary to this, the Rv93 model shows that limited breaking occurs in the trough and inner surf zone.

For the VN cases (plane slope bathymetry) the starting point of wave breaking is shown to be similar in both models, hence similar surf zone widths. Within the surf zone area $Q_{b}$-Rv93 progressively increases toward the shoreline, with maximum values slightly less than 1. $Q_{b}$-ADA increases slightly more rapidly than $Q_{b}$-Rv93 leading to a fully saturated inner surf zone $\left(Q_{b}\right.$-ADA $=$ $1)$. 

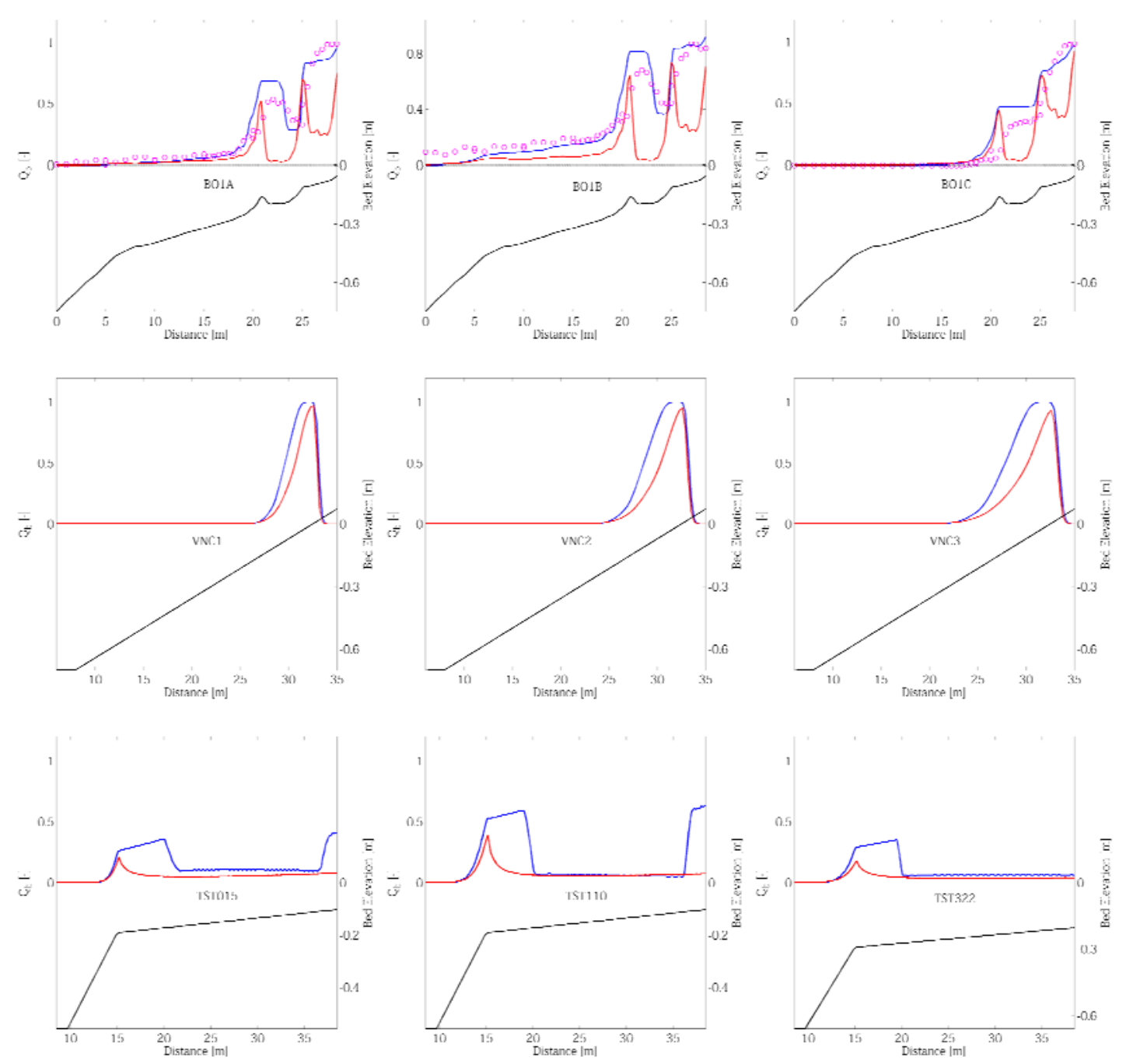

Figure 2: Cross shore variation of the fraction of breaking waves, $Q_{b}$, for the three wave cases of Boers (top row), Van Noorloos (middle row) and Van der Meer (bottom row). Model results obtained using the ADA and Rv93 breaker models are shown as blue and red lines respectively. Measured data (pink circles) is shown where available.

In the VdM wave cases (stepped bathymetry), $Q_{b}$-ADA picks up sharply at the step and continues for some distance over the mild inner slope before reducing. Despite the lack of measurements in the experiment, this result (sustained breaking) is what we would generally expect in nature. The result shown by $Q_{b}$-Rv93 suggests that breaking peaks at the step but is ephemeral, rapidly decreasing after the step. Additionally, $Q_{b}$-ADA indicates that there is a second point of breaking close to the end of the flume (cases TST015 and TST110) which is not shown in the $Q_{b}$-Rv93 results.

\section{Short Wave Height Transformation}

Figure 3 below shows that both $H_{r m s}-\mathrm{ADA}$ and $H_{r m s}$-Rv93 qualitatively represents the measured data quite well for all of the wave cases. In most cases, $H_{r m s}$-ADA in the surf zone is slightly lower than $H_{r m s}-\mathrm{Rv} 93$. This is somewhat attributed to the higher levels of breaking predicted by the ADA breaker model.

In the Boers wave cases $H_{r m s}$-ADA is slightly higher than $H_{r m s}$-Rv93 leading up to the nearshore bar. Results from both breaker models deviate from the measurements in the surf zone, with $H_{r m s}$-ADA and $H_{r m s}-$ Rv93 being relatively lower and higher respectively. Results from $H_{r m s}$-ADA however more closely fit $H_{r m s}$-Meas in the inner surf zone than $H_{r m s}$-Rv93, the latter tending to overestimate $H_{r m s}$.

In the VN cases (plane slope bathymetry) measured and computed results of $H_{r m s}$ are very well matched. $H_{r m s}$-ADA is only marginally less than $H_{r m s}$-Rv93. 

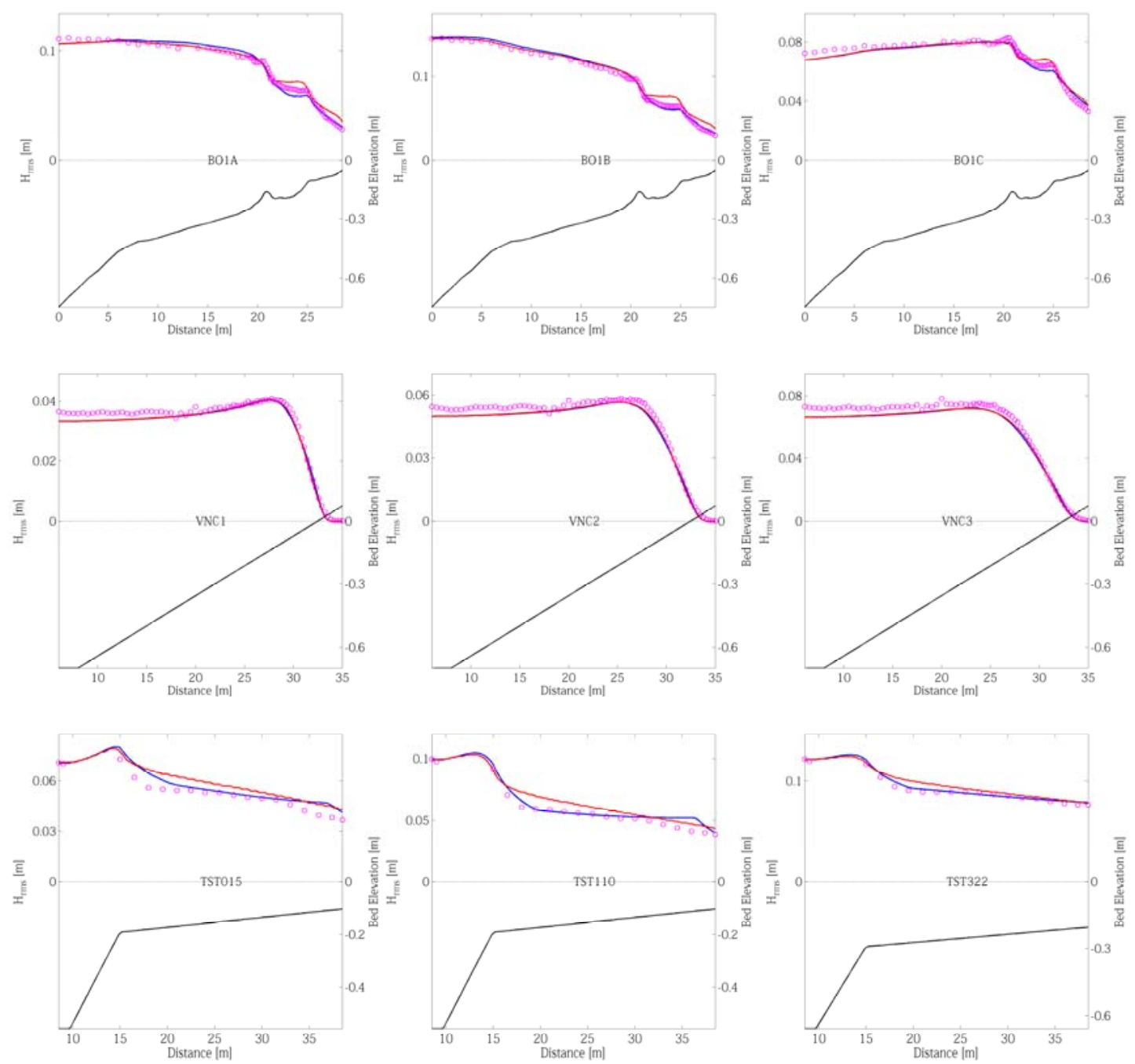

Figure 3: Cross shore variation of the RMS short wave height, $\boldsymbol{H}_{r m s}$, for the three wave cases of Boers (top row), Van Noorloos (middle row) and Van der Meer (bottom row). Model results obtained using the ADA and Rv93 breaker models are shown as blue and red lines respectively. Measured data (pink circles) is shown where available.

For the VdM cases (stepped slope) $H_{r m s}$-Meas shows slight shoaling before the step and a significant decrease height thereafter. Gradual reduction of $H_{r m s}$-Meas occurs as waves propagate over the milder slope. For cases TST015 and TST110 there is a second point where dissipation of $H_{r m s^{-}}$ Meas increases, located toward the end of the flume. Both breaker models capture the initial shoaling and dissipation of $H_{r m}$-Meas, however the rate of decay of $H_{r m s}$-ADA more closely matches $H_{r m}$-Meas than $H_{r m s}$-Rv93. $H_{r m s}$-ADA also shows a second point of increased wave dissipation toward the end of the flume, although the location is different to that indicated by $H_{r m}$-Meas ( $\sim 6 \mathrm{~m}$ difference).

\section{Short Wave Groupiness}

The $G F_{H}$ for all 9 cases considered are shown below in Figure 4. $G F_{H}$-ADA and $G F_{H}$-Rv93 are similar in the initial non-breaking sections of the flume (i.e. locations where $Q_{b}=0$ ). Generally, at the onset of breaking the $G F_{H}$ is consistently reduced towards the shoreline under the influence of wave breaking. The $G F_{H}$, however, recovers within the inner surf zone and above the waterline. In all cases, once wave breaking has begun, the $G F_{H}$-ADA and $G F_{H}$-Rv93 begin to differ.

For the Boers 1A and 1B experiments, the $G F_{H}-\mathrm{ADA}$ is greater than the $G F_{H}-\mathrm{Rv} 93$ leading up to the bar. At the bar, wave breaking becomes more pronounced and results in a significant reduction of $G F_{H^{-}}$ADA until it eventually becomes lower than the $G F_{H^{-}}$Rv 93 values. As the short waves propagate further into the inner surf zone, the $G F_{H^{-}}$ADA begins to recover and is again greater than the $G F_{H^{-}}$ Rv93. For Boers 1C, $G F_{H}$-ADA shows a smoother decrease toward the waterline than $G F_{H}$-Rv93, with some difference within the nearshore trough. 

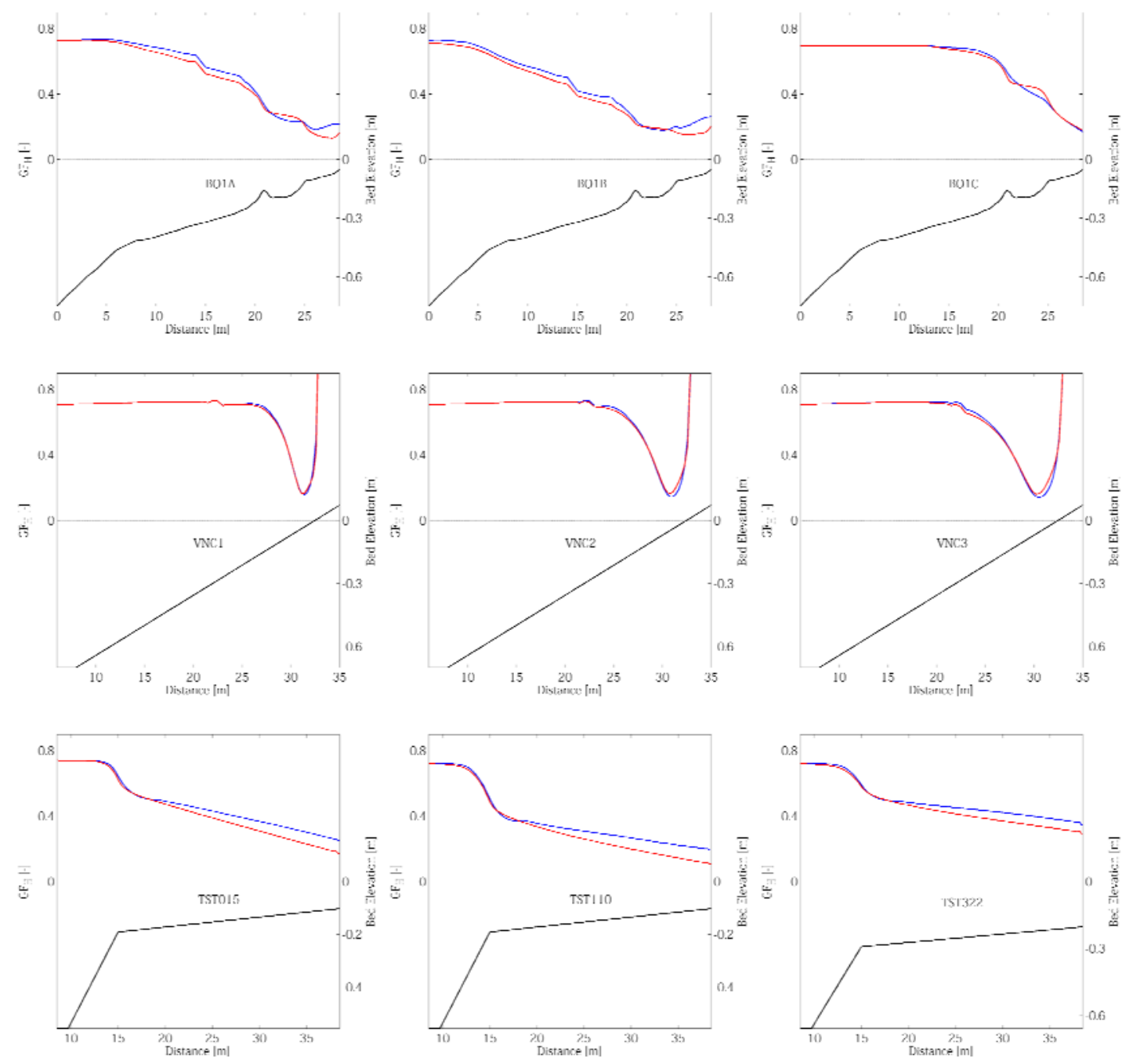

Figure 4: Cross shore variation of the short wave groupiness factor, $G F_{H}$, for the three wave cases of Boers (top row), Van Noorloos (middle row) and Van der Meer (bottom row). Model results obtained using the ADA and Rv93 breaker models are shown as blue and red lines respectively.

Results for the VN experiment indicates that $G F_{H}$-ADA is only slightly higher than $G F_{H}$-Rv93 during breaking, but in general, there hardly any substantial difference between the results for wave propagation over plane slopes.

In the VdM experiments $G F_{H}$ values for both breaker models are similar leading up to the step, however, after the step $G F_{H}$-Rv93 reduces at a faster rate than $G F_{H}$-ADA. Interestingly for these cases, while $G F_{H}$-ADA remains higher than $G F_{H}$-Rv93 the opposite occurs for the $H_{r m s}$ results presented above.

\section{LF Wave Height Transformation}

In general, $\zeta_{r m s}$ increases as the waves propagate into shallow water, as shown in Figure 5 below. It reaches a maximum height at the waterline, and significantly decreases toward the point of maximum run-up (as shown for the plane slope cases of VN). The $\zeta_{\text {rms }}$ shown is a combination of incoming and reflected waves (therefore un-decomposed). $\zeta_{r m s}$-Meas is only available for Boers and VN cases. In these particular cases, the model results generally qualitatively and quantitatively ${ }^{4}$ reflect the measurements, but it is our main interest to note differences between the results from the ADA and Rv93 breaker models.

${ }^{4}$ It should be noted that slight variations in $f_{c u t}$ will affect values of $\zeta_{r m s}$-Meas, as more (or less) energy in included (or excluded) from the LF section of the wave spectrum. 

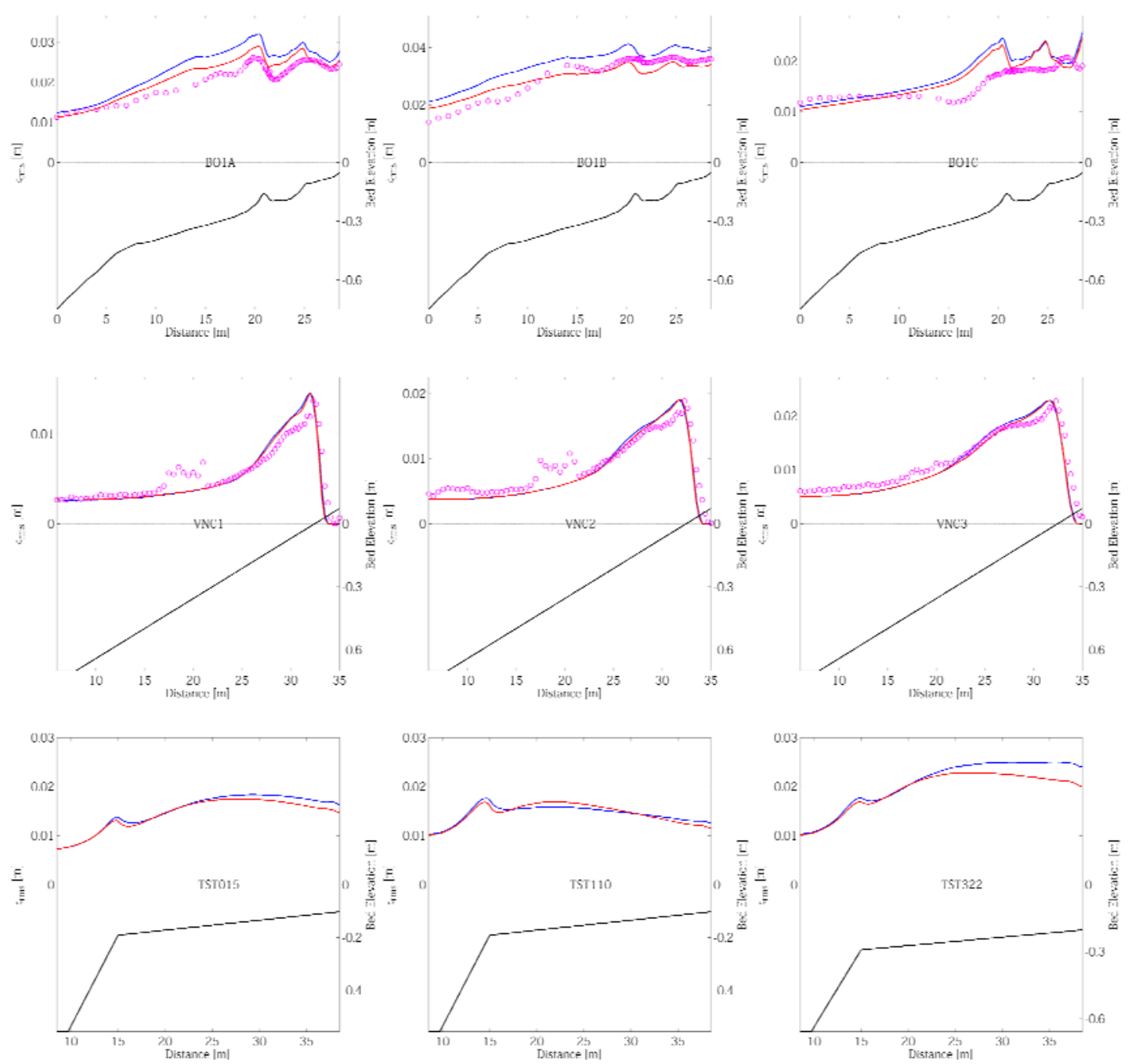

Figure 5: Cross shore variation of the RMS LF wave height, $\zeta_{r m s}$, for the three wave cases of Boers (top row), Van Noorloos (middle row) and Van der Meer (bottom row). Model results obtained using the ADA and Rv93 breaker models are shown as blue and red lines respectively. Measured data (pink circles) is shown where available.

In the Boers experiments, $\zeta_{r m s}$-ADA is constantly higher than $\zeta_{r m s}-\mathrm{Rv} 93$, with differences in the results as high as $17 \%$ (at the nearshore bar). Only in case $1 \mathrm{C}$, where the incoming wave energy is lower, are the model results most similar. Depending on the case, $\zeta_{r m s}$-Meas is either lower than the model results (cases 1A and 1C) or in between them (case 1B).

The results from the VN experiments show only slight difference between $\zeta_{r m s}$-ADA and $\zeta_{r m s}-$ Rv93 in the breaking zone, with no significant difference otherwise. In all cases the model results fit $\zeta_{\text {rms }}$-Meas quite well, even having similar peak values. It should be noted that $\zeta_{r m s}$-Meas for cases C1 and $\mathrm{C} 2$ are affected by spurious recordings in the mid-section of the flume.

Although $H_{r m s}$-ADA is lower than $H_{r m s}-\mathrm{Rv} 93$ for the $\mathrm{VdM}$ experiments, $\zeta_{r m s}$-ADA is generally higher than $\zeta_{r m s}-R v 93$, except for case TST110 where they fluctuate around each other. This illustrates that $\zeta_{\text {rms }}$ is not only dependent on the relative magnitude of $H_{r m s}$. Differences between $\zeta_{r m s}$-ADA and $\zeta_{r m s}-\mathrm{Rv} 93$ are as high as $20 \%$ at the upper end of the flume (case TST322).

\section{Wave Set-down and Set-up}

Time-averaged low frequency motions show the variation in the set-down and set-up of waves in the nearshore (the cross-shore $M W L$, illustrated in Figure 6). The $M W L$ is generally lowered under shoaling waves as their heights increase, but it begins to rise as wave breaking dissipates excess wave energy. The $M W L$ is therefore dependent on the local bathymetry which controls the areas where breaking will occur. 

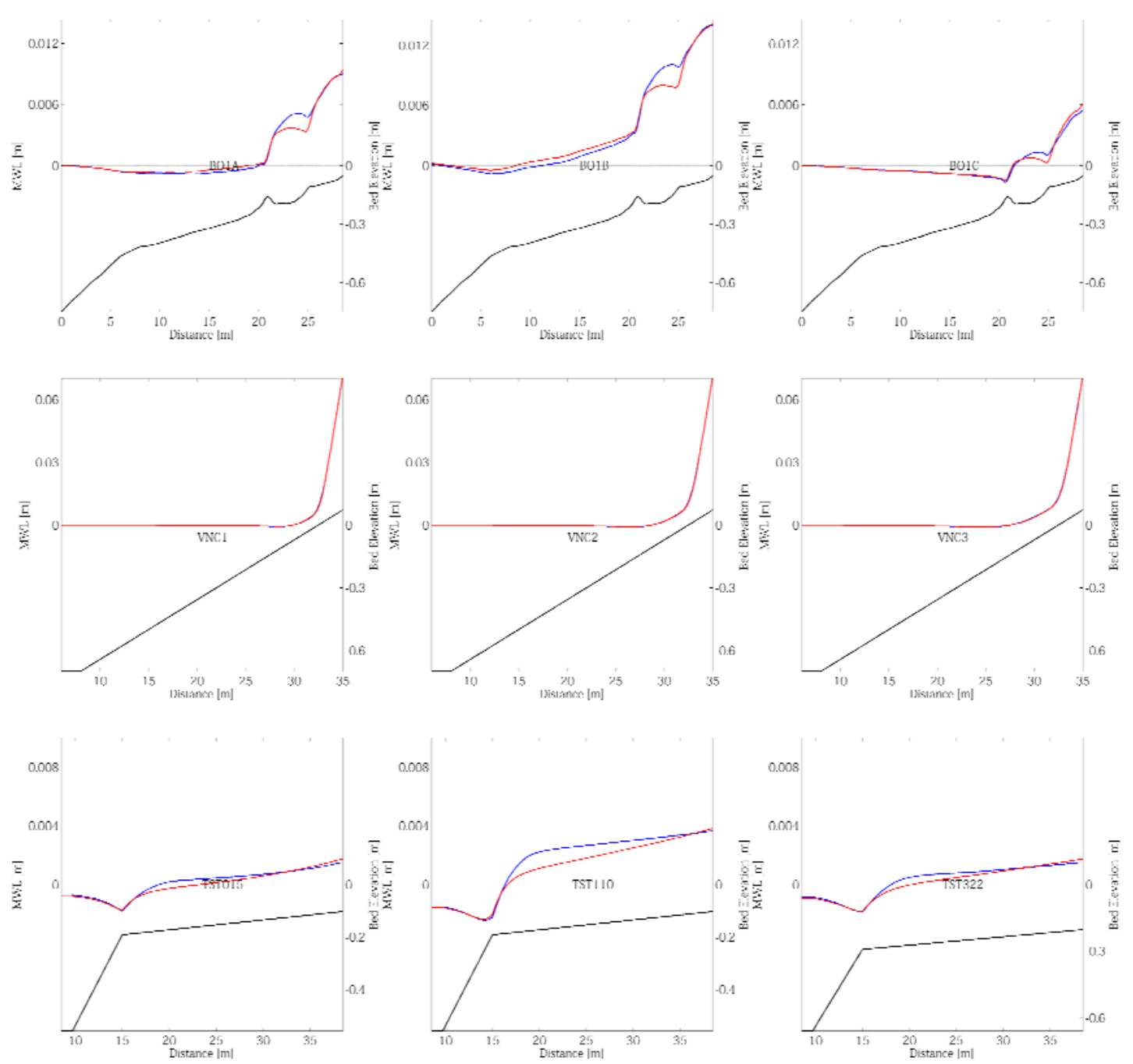

Figure 6: Cross shore variation of the mean water level, $M W L$, for the three wave cases of Boers (top row), Van Noorloos (middle row) and Van der Meer (bottom row). Model results obtained using the ADA and Rv93 breaker models are shown as blue and red lines respectively.

For the Boers wave cases the set-down and set-up of the $M W L-\mathrm{ADA}$ is greater than that shown by $M W L-R v 93$ in wave breaking areas of the flume. The difference in set-up values predicted by both breaker models is greatest within the nearshore trough, exceeding $20 \%$ for case $1 \mathrm{~B}$ for example. In the inner surf zone, however, $M W L-\mathrm{ADA}$ and $M W L-\mathrm{Rv} 93$ are similar, which also leads to similar maximum set-up values in the swash zone.

As shown with the previous variables, there is also no significant difference between $M W L$-ADA and $M W L-\mathrm{Rv} 93$ for the wave cases of $\mathrm{VN}$ featuring a plane slope. The maximum set-up predicted by both models in the swash zone is similar.

There is an initial set-down in the VdM experiments; however, wave set-up starts immediately after the step in the bathymetry. The $M W L$-ADA climbs to considerably higher values than $M W L$-Rv 93 following the step. Comparing Figure 6 to Figure 2, the sharp rise of $M W L$-ADA is commensurate with the area of heightened and sustained wave breaking as predicted by the ADA breaker model.

\section{DISCUSSION}

The results presented above highlight some of the implications which arise when using the ADA breaker model to determine short wave breaking. Results for $\zeta_{r m s}$ and $M W L$ varied for each of the three bathymetric cases. As described in the numerical model section above, computed $\zeta_{\text {rms }}$ are determined by radiation stress gradients in the shoaling and breaking zone. These gradients are affected by the magnitude and level of variance (or groupiness) of the short waves. $\zeta_{r m s}$ will therefore be higher during higher energy wave conditions than during lower energy wave conditions. Also, higher levels of 
groupiness of the short waves will tend to yields higher $\zeta_{r m s}$. This can be seen in the results presented above (and which is highlighted again in this discussion). Additionally, it is known that the forcing of LF waves by short waves (which are in anti-phase with each other during shoaling) stops during wave breaking as their phases become positively correlated (Van Dongeren et al., 2007). This phenomenon plays and important role in explaining the results obtained. In this discussion, the results will be used to highlight the effect of short wave breaking on LF waves on a case by case basis with a summary of the findings at the end.

\section{Wave Cases}

For the simpler plane slope bathymetric cases (VN experiments), predictions of $H_{r m s}$ and $G F_{H}$ from the ADA and Rv93 breaker models show only slight differences. Since wave breaking begins at the same location in the flume using both breaker models, with constant dissipation of the wave height thereafter toward the shoreline, short wave forcing is fairly similar which also leads to similar predictions for $\zeta_{r m s}$ and $M W L$. Increased incoming wave energy (case $\mathrm{C} 1$ being lowest and case $\mathrm{C} 3$ being highest) also results in increased $\zeta_{\text {rms }}$ and $M W L$ peak values.

The remaining experiments (Boers and VdM) features more complex bathymetry. Results from these cases point out differences in $H_{r m s}, G F_{H}, \zeta_{r m s}$ and $M W L$ predictions coming from the ADA and Rv93 breaker models. These differences are related to the method in which wave breaking is schematized in the two models. It is shown that the ADA breaker model predicts more prolonged wave breaking after sudden changes in bathymetry (at nearshore bars and steps) than the Rv93 breaker model. The difference in the results for $Q_{b}$ propagates through the model to further affect $H_{r m s}$ and $G F_{H}$ and ultimately $\zeta_{\text {rms }}$ and $M W L$.

In the Boers experiment, $H_{r m s}$-ADA is slightly higher than $H_{r m s}$-Rv93 in the area leading up to the nearshore bar and also, to a greater degree, $G F_{H}$-ADA is greater than $G F_{H}$-Rv93. This combination leads to greater forcing of the $\zeta_{r m s}$ using the ADA breaker model as illustrated in Figure 5. $M W L$-ADA predictions are significantly higher after the nearshore bar than $M W L-R v 93$, owing to increased wave breaking in the area predicted by the ADA breaker model.

For the $\mathrm{VdM}$ experiments, $H_{r m s}$ and $G F_{H}$ results (and thus $\zeta_{r m s}$ ) are initially similar for both breaker models up to a short distance beyond the step in the bathymetry $(\sim 3 \mathrm{~m})$. Thereafter, $H_{r m s}$-ADA becomes lower than $H_{r m s}$-Rv93 due to prolonged wave breaking in the ADA breaker model, however, $G F_{H}$-ADA remains higher than $G F_{H}$-Rv93. Despite being initially similar to $\zeta_{r m s}$-Rv93, $\zeta_{r m s}$-ADA gradually increases in height over $\zeta_{r m s}$-Rv93 by the end of flume. This is mainly attributed to the higher $G F_{H}$-ADA results post-breaking at the step, as the magnitude of $H_{r m s}$-ADA is lower than $H_{r m s}$-Rv93. Similar to the Boers wave cases, the $M W L$-ADA predictions are significantly higher than $M W L$-Rv93 after the step in the flume, but both climb to similar values at the end of the flume.

\section{Summary}

Radiation stress gradients over wave groups are derived from the magnitude of short wave heights and additionally from the groupiness of the short waves. The combination of these sources primarily forces LF waves. Given that the short wave timeseries is regulated by dissipation through wave breaking, the type of breaking mechanism employed becomes important in the prediction of LF waves. The underlying bathymetry is shown to influence wave breaking and thus wave groupiness. For a simple, plane slope the difference in forcing for both breaker models is negligible and similar results for LF wave heights and water levels are obtained.

However, complex bathymetries can impose arbitrary wave breaking locations such as at nearshore bars or steps (analogous to coral reefs in nature). Since the ADA model tends to sustain breaking until waves become stable, upwind variations of the short wave height are different from the Rv93 model for these cases. The ADA breaker model produces a short wave timeseries with greater variance than the Rv93 breaker model. This is indicative of a pulsating nature of wave breaking predicted by the former, a characteristic which is obtained by the deterministic manner in which wave breaking is switched on and off. On the other hand, the probabilistic function in the Rv93 formulation tends to smoothen wave breaking over a wider area.

The greater short wave variance present in the short wave timeseries from ADA breaker model gives additional forcing to LF waves. The effect is even more pronounced during more energetic wave conditions, for example in cases Boers-1B and VdM-TST322 where the relative difference between $\zeta_{r m s}$-ADA and $\zeta_{r m s}$-Rv93 is up to $20 \%$. Prolonged wave dissipation after wave-breaking-inducing bathymetric features as predicted by the ADA breaker model results in significantly greater $(>25 \%)$ 
wave set-up behind (and potentially greater flow over) these features than predicted by the Rv93 breaker model.

\section{CONCLUSION}

The method in which wave breaking is characterized can affect the propagation of short waves over complex bathymetry and thereby influence LF waves via changes in radiation stress gradients. The accurate prediction of $Q_{b}$ and LF waves is important as they determine wave set-up and can affect flows in the nearshore.

Moderate quantitative difference ( $>15 \%$ ) between results from the ADA versus the Rv93 breaker model for LF wave heights is obtained. This is generally because the ADA model allows higher levels of short wave groupiness to remain in the surf zone. The difference between the ADA and Rv93 breaker model results are even greater for wave set-up ( $>25 \%)$, which is attributed to greater levels of breaking after sudden changes in bathymetry. The effect is amplified for greater levels of incident wave energy.

\section{ACKNOWLEDGMENTS}

The Research reported in this document has been made possible through the support of the European Community's Seventh Framework Program under grant agreement no. 202798 (MICORE Project) and Deltares internal research fund under Strategic Research project 1200266.

\section{REFERENCES}

Battjes, J.A., Bakkenes, H.J., Janssen, T.T., van Dongeren, A.R. 2004. Shoaling of sub-harmonic gravity waves, J. Geophysical Research, 109, C02009, doi:10.1029/2003JC001863.

Boers, M. 1996. Simulation of a surf zone with a barred beach; Report 1: Wave heights and wave breaking, Communication on Hydraulic and Geologic Engineering, Delft University of Technology, No. 96-05.

Dally, W.R. 1990. Random breaking waves: a closed-form solution for planar beaches, J. Coastal Engineering, 14, 233-263.

Dally, W.R., R.G. Dean and R.A. Dalrymple. 1984. A model for breaker decay on beaches, Proceedings of $19^{\text {th }}$ International Conference on Coastal Engineering, New York, ASCE, 82-98.

Daly, C.J., J.A. Roelvink, A.R. van Dongeren, J.S.M. van Thiel de Vries, R.T. McCall. In Review. Evaluation of an advective-deterministic approach to wave breaking, J. Coastal Engineering.

Deigaard, R. 1993. A note on the three dimensional shear stress distribution in a surf zone, J. Coastal Engineering, 20, 157-171.

List, J.H. 1992. Breakpoint-forced and bound long waves in the nearshore: A model comparison, Proceedings of $23^{\text {rd }}$ International Conference on Coastal Engineering, Venice, ASCE, 860-873

Longuet-Higgins, M.S., and R.W. Stewart. 1962. Radiation stress and mass transport in gravity waves, with application to 'surf beats', J. Fluid Mechanics, 13, 481-504.

Munk, W.H. 1949. Surf beats, Trans. American Geophysical Union, 30, 849-54.

Raubenheimer, B. and R. T. Guza. 1996. Observations and predictions of run-up, J. Geophysical Resarch, 101(C11), 25, 575-587.

Roelvink, J.A. 1993. Dissipation in random wave groups incident on a beach, J. Coastal Engineering, $19,127-150$.

Roelvink, J.A., Reniers, A.J.H.M. 1995. LIP 11D delta flume experiments, Data Report H2130, Delft Hydraulics, Delft.

Roelvink, J.A., A.J. Reniers, A.R. van Dongeren, J.S.M. van Thiel de Vries, R. McCall and J. Lescinski. 2009. Modeling storm impacts on beaches, dunes and barrier islands, J. Coastal Engineering, doi:10.1016/ j.coastaleng.2009.08.006.

Schäffer, H.A. and I.A. Svendsen. 1988. Surf beat generation on a mild slope beach, Proceedings of $21^{\text {st }}$ International Conference on Coastal Engineering, ASCE, 1058-1072.

Schäffer, H.A. 1993. Infragravity waves induced by short-wave groups, J. Fluid Mechanics, 247, 551588.

Symonds, G., D.A. Huntley and A.J. Bowen. 1982. Two-dimensional surf beat: Long wave generation by a varying breakpoint, J. Geophysical Research, 80, 492-498.

van der Meer, J.W. 1990. Golfhoogtes van brekende golven op een flauw talud (Wave heights for breaking waves on a flat slope, in Dutch), Delft Hydraulics report no. H462-IV, Delft. 
van Dongeren, A.R., J.A. Battjes, T.T. Janssen, J.C. van Noorloos, K. Steenhauer, G. Steenbergen and A. Reniers. 2007. Shoaling and shoreline dissipation of low-frequency waves, J. Geophysical Research, 112, C02011, doi:10.1029/2006 JC003701.

van Noorloos, J.C. 2003. Energy transfer between short wave groups and bound long waves on a plane slope, M.Sc. Thesis, Delft University of Technology.

van Thiel de Vries, J.S.M., J. van de Graaf, B. Raubenheimer, A.J. Reniers and M.J.F. Stive. 2006. Modelling inner surf hydrodynamics during storm surges, Proceedings of $30^{\text {th }}$ International Conference on Coastal Engineering, San Diego, ASCE, 896-908. 